\title{
Características biosociales, reproductivas y obstétricas asociadas al resultado adecuado del parto en Ica, Perú, 2013
}

\author{
Oscar Munares-García*
}

\begin{abstract}
*Licenciado en Obstetricia. Magister en Salud Pública. Doctor en Ciencias de la Salud. Departamento Académico de Obstetricia. Facultad de Medicina Humana San Fernando. Universidad Nacional Mayor de San Marcos. Instituto Nacional de Salud. Lima. Perú
\end{abstract}

Correspondencia: Dr. Oscar Munares García. Dirección: Av. Tizón y Bueno 276 Lima 11, Perú. Correo electrónico: omunaresg@unmsm.edu.pe

\section{RESUMEN}

Introducción: El llevar la salud materna a los hospitales ha enfocado al parto como problema de salud, es necesario estudiarlo como proceso normal e incorporar prácticas adecuadas. Objetivo: Determinar las características biosociales, reproductivas y obstétricas asociadas al resultado adecuado del parto. Materiales y métodos: Estudio analítico transversal realizado en 338 mujeres embarazadas procedentes del Hospital IV Augusto Hernández de Seguro Social de Salud de Ica, Perú entre marzo y abril de 2013, quienes fueron divididas en dos grupos según el resultado del parto: adecuado $(n=174)$ e inadecuado $(n=164)$. Se consideró resultado adecuado si el parto fue por vía vaginal, no presentó desgarros perineales, el líquido amniótico fue claro, el recién nacido tuvo un APGAR al minuto mayor a siete, la edad gestacional estuvo entre 37 a 41 semanas y el peso al nacer entre los 2501 a 3999 gramos. Se aplicaron estadísticas descriptivas, Odds Ratio con IC 95\% y regresión logística para asociaciones. Resultados: El 78,4\% de las gestantes tuvo entre 20 a 35 años, el 51,6\% presentó resultado adecuado del parto. El tener una edad entre 20 a 35 años (ORa 0,4 IC95\% 0,2-0,7), nivel educativo secundario o superior (ORa 4,1 IC95\% 1,2-13,8), más de cuatro controles prenatales (ORa 2,9 IC 95\% 1,4-6,3) y haber participado en sesiones de psicoprofilaxis obstétrica (ORa 1,7 IC95\% 1,03-2,7) se asociaron a un resultado adecuado del parto. Los factores reproductivos como menarquia antes de los 12 años y el uso de anticonceptivo no mostraron asociación. Conclusiones: Alrededor de la mitad de las gestantes tienen un resultado adecuado del parto. Los prestadores de atención materna deberían incorporar la edad, nivel educativo secundaria o superior, controles prenatales mayores de cuatro y la psicoprofilaxis como indicadores de resultado. MÉD UIS. 2015;28(3):291-9.

Palabras clave: Parto humanizado. Atención prenatal. Parto Obstétrico. Educación prenatal. Embarazo.

\section{Biosocial, reproductive and obstetric characteristics associated with adequate result of delivery in Ica, Peru, 2013}

\begin{abstract}
Introduction: The make maternal health in hospitals, it has focused on delivery as a health problem, but it is necessary to study it as a normal process with incorporating best practices. Objective: Determine the social, reproductive and obstetric characteristics associated with adequate birth outcomes. Material and methods: Analytic cross-sectional study, in 338 births from the Hospital IV Augusto Hernandez EsSalud from Ica, Peru, between March and April 2013, which were divided into 174 with adequate of delivery result and 164 with inadequate result of delivery. Adequate result of delivery was considered a vaginal birth without perineal tears, with clear amniotic fluid and newborn with Apgar 7 to minute, gestational age between 37-41 weeks and birth weight between 2501-3999 grams. Descriptive statistics were applied, odds ratio (OR) with $95 \%$ and logistic regression for associations (aOR). Results: $78.4 \%$ of pregnant women had between 20-35 years, 51.6\% had adequate result of delivery. Having aged between 20-35 years (aOR: 0.4 Cl95\% 0.2-0.7), secondary or higher education (aOR: $4.1 \mathrm{Cl} 95 \%$ 1.2-13.8), prenatal care >4 (aOR: $2.9 \mathrm{Cl} 95 \%$ 1.4-6.3) and have participated in sessions of obstetrical psycho (aOR: $1.7 \mathrm{Cl}$ 95\% 1.03-2.7) are associated with adequate result of delivery. Reproductive factors such as age at menarche $<12$ years and contraceptive use showed no association. Conclusions: About half of pregnant women have adequate results of delivery. Maternity care providers should incorporate age, secondary or higher education, prenatal care $>4$ and Psychoprophylactic as appropriate outcome about adequate result of delivery. MÉD UIS. 2015;28(3):291-9.
\end{abstract}

Keywords: Humanizing Delivery. Prenatal care. Delivery. Obstetric. Prenatal Education. Pregnancy. 
¿Cómo citar este artículo?: Munares-García O. Características biosociales, reproductivas y obstétricas asociadas al resultado adecuado del parto en Ica, Perú, 2013. MÉD UIS. 2015;28(3):291-9.

\section{INTRODUCCIÓN}

El parto es un suceso importante en la familia, por ello debe de ser participativo, seguro y culturalmente aceptado. Existen dos modos de poder visualizar la salud materna, uno enfocado en los problemas para poder corregirlos, y el otro basado en la promoción de la salud y el autocuidado. El sistema de salud en el Perú se realiza por subsectores, prestadores de servicios de salud como el Ministerio de Salud, seguro social de salud, sanidades de las fuerzas armadas y policiales y las entidades privadas'. La estrategia de mejora de la salud materna en el Perú, se enfoca en la atención prenatal por personal calificado y parto institucional en todas las gestantes. En la región de Ica según la Encuesta Demográfica y de Salud Familiar (ENDES) determinó que para el año 2012 el 99,4\% de las mujeres entre los 15 a 49 años recibió atención prenatal por parte de un médico, obstetra o enfermera y el $98,7 \%$ de los partos ocurrieron en un establecimiento de salud ${ }^{2}$. En este mismo lugar para el 2011, el Seguro Social de Salud (EsSalud) cubrió al $28,1 \%$ de la población 3 .

Para que un parto presente menores problemas, este debe darse en condiciones adecuadas, iniciando con un embarazo planificado, a una edad, convivencia y nivel educativo apropiado; así mismo debe tener un número mínimo de controles prenatales y sesiones de psicoprofilaxis obstétrica. El parto debe de realizarse con acompañante, preferiblemente por vía vaginal y se espera que el líquido amniótico sea claro, no se presenten desgarros perineales y el bebé nazca con una edad gestacional entre las 37 a 41 semanas, con un peso entre los 2501 a 3999 gramos y un puntaje de Apgar mayor a siete. La Organización Mundial de la Salud (OMS) define el "parto normal", como aquel que comienza espontáneamente, tiene bajo riesgo desde el inicio hasta el alumbramiento, el niño nace en posición cefálica entre las 37 a 42 semanas y después de dar a luz, tanto la madre como el niño, se encuentran en buenas condiciones ${ }^{4}$.

Para ello, existen una serie de factores biosociales, reproductivos y obstétricos que se asocian al resultado adecuado del parto. Entre los factores biosociales está la edad, que es considerada de bajo riesgo si se encuentra entre los 20 a 35 años. A la adolescencia se le ha asociado problemas biológicos, psicológicos y sociales, teniendo mayor riesgo de anemia, hipertensión inducida por el embarazo, diabetes, parto distócico, hemorragias, recién nacido con bajo peso, enfermedades genéticas, alteraciones psicosociales y crisis familiar 5 . Por otro lado, la gestante mayor de 35 años se le ha relacionado hipertensión arterial inducida por el embarazo, (RR: 1,9) diabetes gestacional (RR: 3,7), amenaza de parto prematuro (RR: 4,7 ) y metrorragia en el primer trimestre (RR: 2,9) ${ }^{6}$.

Con respecto al estado civil, existe mayor probabilidad que las gestantes casadas o de unión libre (convivientes) presenten más probabilidad de embarazo planificado, y por ende mayor posibilidad de llevar un embarazo y parto adecuado comparada con las que son solteras o separadas. Con respecto al nivel educativo se ha demostrado que el riesgo de muerte neonatal precoz es mayor en gestantes analfabetas (OR: 2,9 IC95\% 1,02:8,4)7. En cuanto a la ocupación de la madre, se sabe que las gestantes que trabajan tienen mejores y mayores atenciones maternas que aquellas que no trabajan ${ }^{8}$.

Entre las características reproductivas esta la menarquia precoz y los métodos anticonceptivos. Se considera que las mujeres con menarquia precoz (antes de los 12 años) tienen mayor probabilidad de embarazos a edades más tempranas ${ }^{9}$. Así mismo, las mujeres que no emplean métodos anticonceptivos tienen un mayor riesgo de embarazo no planificado ${ }^{5}$ que puede llevar a malos resultados en el embarazo y el parto.

Entre las características obstétricas se encuentra el control prenatal, antecedente de aborto, paridad, psicoprofilaxis obstétrica y apoyo del acompañante en el parto. Se conoce que el control prenatal reduce la morbimortalidad materna y perinatal, el número de partos prematuros y el bajo peso al nacer. De igual forma permite identificar factores de riesgo, lo cual hace posible establecer acciones preventivas y terapéuticas oportunas durante el embarazo ${ }^{10}$. La Organización Mundial de la Salud establece que las embarazadas deben asistir mínimo a cinco controles prenatales considerado adecuado", en Perú es con seis controles ${ }^{12}$.

La psicoprofilaxis obstétrica o parto indoloro psicoprofiláctico prepara a la mujer mental y 
físicamente para el embarazo y parto, permite desarrollar hábitos y comportamientos saludables así como una actitud positiva frente al embarazo, parto, puerperio y concepción del recién nacido ${ }^{13,14}$. Es un elemento de mejora de la calidad de vida de la gestante y la familia ${ }^{15}$, en la norma peruana tiene que seis o más sesiones son necesarias para considerar a una gestante preparada ${ }^{16}$. El parto con acompañante forma parte del parto humanizado ${ }^{4}$, complementa la atención médica y mejora aspectos psicoafectivos y culturales ${ }^{17}$.

Otro elemento obstétrico característico es la paridad, se ha estudiado que los patrones fisiológicos son diferentes en la nulípara que en la multípara, tanto en el trabajo de parto como en el expulsivo ${ }^{18}$. Finalmente, el antecedente de aborto, también juega un rol importante en el parto, se sabe que los legrados uterinos pueden condicionar cicatrices que pueden llevar a casos de placenta previa.

El enfoque referente al parto humanizado se centra, más que en las condiciones de riesgo de una gestante, en un enfoque centrado en los elementos que llevan a un parto digno con participación de la familia4. Por ello las variables sociales como la edad, el nivel educativo, la religión, así como los esfuerzos preventivos que realiza el sistema de salud en este ámbito como el control prenatal, la preparación psicoprofiláctica $^{14}$ y la estimulación prenatal ${ }^{16}$, se centran en un resultado adecuado del parto. Por lo expuesto anteriormente, el objetivo de este trabajo fue determinar las características sociales, reproductivas y obstétricas asociadas al resultado adecuado del parto.

\section{Materiales y Métodos}

Se realizó un estudio transversal analítico. Se tomaron datos registrados en historias clínicas y se confeccionó una hoja de registros para el ingreso de datos. Por tratarse de un estudio retrospectivo con evaluación de las historias clínicas, no se registraron datos de identificación como los nombres y números de identificación de gestantes o sus acompañantes, por lo que se manejó la información vía códigos. Se tomaron los datos referentes a la edad, estado civil, ocupación, edad de la menarquia, edad de inicio de relaciones sexuales, número de gestaciones, controles prenatales, peso del recién nacido y edad gestacional, de todas las gestantes atendidas en el Hospital IV Augusto Hernández Mendoza de EsSalud de la ciudad de Ica, Perú, en el periodo de marzo a abril de 2013 que presentaron parto vaginal y recién nacidos vivos; y se excluyeron gestaciones múltiples. En total fueron 338 gestantes quienes se dividieron en dos grupos: 174 con resultado adecuado del parto y 164 con resultado inadecuado del parto.

Se definió resultado adecuado del parto cuando no hubo desgarros perineales, el recién nacido nació por parto vaginal, tuvo un índice de Apgar al minuto mayor de siete, peso entre 2501 a 3999 gramos, el líquido amniótico fue claro y la edad gestacional estuvo entre las 37 a 41 semanas ${ }^{12,19,20}$, aquellos que no cumplieron con todos los requisitos antes indicados se consideró resultado inadecuado del parto. En algunas variables se procedió a una mayor especificación, como en la edad, si era menor de 19 años o mayor de los 35 años, si la escolaridad era de nivel primario, nivel secundario o superior y el estado civil. Las características reproductivas fueron menarquia antes de los 12 años y uso de método anticonceptivo. Las características obstétricas fueron primigestante, control prenatal mayor o igual a cuatro controles, haber tenido sesiones de psicoprofilaxis obstétrica y apoyo de pareja.

Se solicitó aprobación por la dirección del departamento, se acudió al área de hospitalización, donde se revisó las historias clínicas de las pacientes, luego se tomaron los datos del parto en el libro de centro obstétrico, y la historia clínica del recién nacido, cuando hizo falta algún dato se consultó directamente a la gestante. La información se procesó en una base de datos en IBM-SPSS versión 20.0.

Para la determinación del resultado adecuado del parto, se confeccionó una sintaxis en SPSS que incluyera todos los requisitos para que luego el software realizará la clasificación entre los que cumplían o no. Se aplicó medidas de tendencia central, percentiles y prueba de KolmorogovSmirnov para la determinación de la normalidad, siendo significativo $p>0,05$. Los datos fueron ponderados de acuerdo a las gestantes procedentes de los distritos de Ica. Para las variables categóricas se aplicó distribución de frecuencias y porcentajes. Para la determinación de las características asociadas, se aplicó análisis bivariado con Chi cuadrado de Pearson o Exacto de Fisher con un nivel de significación $p<$ 0,05, así mismo se estimaron Odds Ratio (OR Crudo) con sus intervalos de confianza al 95\% (IC95\%). Para la determinación del modelo de características asociadas al resultado adecuado del parto, se aplicó regresión logística para la determinación de los OR 
ajustados (ORa) con el método introducir, así mismo se aplicó la prueba de Hosmer y Lemeshow para la evaluación de la calidad y la regresión de Cox y Snell para la determinación del porcentaje explicativo, así como la sensibilidad global del modelo.

\section{ResULTADOS}

Las características biosociales mostraron que la mayoría de las gestantes tenían entre 20 a 35 años (78,4\%), el 51,6\% presentó nivel educativo secundario, el $60,1 \%$ eran convivientes, el $42,9 \%$ fue ama de casa y el 34,6\% era empleada (Ver Tabla 1). Todos los datos no presentaron una distribución normal, la mediana de edad fue de 28 años, la edad de la menarquia fue 13 años, la edad de inicio de relaciones sexuales fue 18 años, el número de gestaciones fue dos y el número de controles prenatales fue siete. La mediana del peso del recién nacido fue 3520 gramos y la edad gestacional fue 40 semanas (Ver Tabla 2).

Tabla 1. Características biosociales de las pacientes atendidas

\begin{tabular}{|c|c|c|}
\hline & $\mathbf{n}$ & $\%$ \\
\hline \multicolumn{3}{|l|}{ Edad (años) } \\
\hline 15 a 19 & 17 & 5,2 \\
\hline 20 a 35 & 265 & 78,4 \\
\hline 36 a 43 & 55 & 16,4 \\
\hline \multicolumn{3}{|l|}{ Nivel educativo } \\
\hline Primaria & 16 & 4,7 \\
\hline Secundaria & 174 & 51,6 \\
\hline Superior & 148 & 43,7 \\
\hline \multicolumn{3}{|l|}{ Estado Civil } \\
\hline Conviviente & 203 & 60,1 \\
\hline Casada & 121 & 35,9 \\
\hline Soltera & 14 & 4,0 \\
\hline \multicolumn{3}{|l|}{ Ocupación } \\
\hline Ama de casa & 145 & 42,9 \\
\hline Empleada & 117 & 34,6 \\
\hline Profesional & 49 & 14,5 \\
\hline Estudiante & 27 & 8,0 \\
\hline Total & 338 & 100,0 \\
\hline
\end{tabular}

Fuente: Autor.

De las pacientes estudiadas, el $95,9 \%$ se encontraban entre las 37 a 41 semanas, el 75,4\% presentó líquido amniótico claro, el $92,6 \%$ no presentó desgarros perineales, todos tuvieron un índice de Apgar al minuto mayor o igual de siete, siendo 9 puntos el más frecuente $(85,5 \%$ ) y el $84,6 \%$ tuvo un recién nacido con peso entre los 2501 a 3999 gramos. Al evaluar los resultados inadecuados del parto, el 8,5\% presentó edades gestacionales menores a 37 semanas, en el $50,6 \%$ se observó líquido amniótico meconial, el 15,2\% tuvo desgarros de primero o segundo grado, el 1,8\% presentó índice de Apgar al minuto igual a 7, y el 31,7\% de los recién nacidos tenía pesos menores a 2500 o mayores a 3999 gramos (Ver Tabla 3).

El 51,6\% tuvo un resultado adecuado del parto, este fue más frecuente en las pacientes que tenían un nivel educativo secundario o superior $(p=0,028)$, controles prenatales mayores a cuatro $(p=0,005)$ y psicoprofilaxis obstétrica $(p=0,009)$. Mientras que al resultado inadecuado del parto fue más frecuente en mujeres que tenían entre 20 a 35 años ( $p=0,005)$. El estado civil conviviente o casada, el ser ama de casa, la menarquia precoz, el uso de método anticonceptivo antes del embarazo y el antecedente de aborto no fueron significativos (Ver Tabla 4).

En el análisis bivariado entre las características biosociales, se encontró asociación para el resultado adecuado del parto a la edad entre 20 a 35 años (OR 0,5 IC $95 \%$ 0,3-0,8) y nivel educativo secundario o superior (OR 3,4 IC95\%1,1-10,8). El ser Conviviente o casada y ama de casa no se asociaron. En cuanto a las características reproductivas, no se encontró asociación con la menarquia y el uso de anticonceptivos antes del embarazo. Entre las características obstétricas se encontró asociación el tener más de cuatro controles prenatales (OR 2,8 IC95\% 1,3-5,9) y la psicoprofilaxis obstétrica (OR 1,9 IC95\% 1,2-2,9), no se encontró asociación con el antecedente de parto, de aborto y apoyo del acompañante.

En el análisis multivariado se encontró relación entre el resultado adecuado del parto con la edad entre 20 a 35 años (ORa 0,4 IC95\% 0,2-0,7), el nivel educativo superior (ORa 4,1 IC 95\% 1,2 - 13,8), controles prenatales mayores a cuatro (ORa 2,9 IC95\% 1,4 - 6,3) y psicoprofilaxis obstétrica (ORa 1,7 IC95\% 1,03-2,7), sin embargo no se halló asociación con las características reproductivas. El tener un nivel educativo secundario o superior incrementa la probabilidad de resultado adecuado del parto en cuatro veces, el control prenatal mayor a cuatro lo incrementa en 2,9 veces, la psicoprofilaxis obstétrica lo aumenta en 1,7 veces y el no encontrarse entre 20 a 35 años incrementa en $60 \%$ el resultado adecuado del parto, estas variables explican el $8,1 \%$ de la varianza del resultado adecuado del parto, tal como lo demuestra el $R^{2}$ Cox y Snell= 0,081, así mismo el modelo resultó adecuado a través de la prueba de Hosmer y Lemeshow $(p=0,877)$ (Ver Tabla 4). 
Tabla 2. Descripción estadística según características obstétricas de las pacientes

Fuente: Autor

\begin{tabular}{|l|c|c|c|c|c|c|c|}
\hline & $\mathbf{n}$ & Media & $\mathbf{D E}$ & $\mathbf{P}_{\mathbf{2 5}}$ & $\mathbf{P}_{50}$ & $\mathbf{P}_{75}$ & $\boldsymbol{p}$ \\
\hline Edad (años) & 338 & 28,2 & 6,0 & 23 & 28 & 33 & $<0,001$ \\
\hline Menarquia (años) & 338 & 13,2 & 1,5 & 12 & 13 & 14 & $<0,001$ \\
\hline $\begin{array}{l}\text { Inicio de relaciones } \\
\text { sexuales (años) }\end{array}$ & 338 & 18,1 & 2,4 & 16 & 18 & 19 & $<0,001$ \\
\hline Gestaciones & 338 & 2,5 & 1,4 & 1 & 2 & 3 & $<0,001$ \\
\hline Controles prenatales & 338 & 6,4 & 2,0 & 5 & 7 & 8 & $<0,001$ \\
\hline $\begin{array}{l}\text { Peso del recién } \\
\text { nacido (g) }\end{array}$ & 338 & 3431,5 & 481,4 & 3110 & 3520 & 3700 & $<0,001$ \\
\hline $\begin{array}{l}\text { Edad gestacional } \\
\text { (semanas) }\end{array}$ & 338 & 39,2 & 1,7 & 39 & 40 & 40 & $<0,001$ \\
\hline
\end{tabular}

Tabla 3. Distribución de las pacientes según resultado del parto

\begin{tabular}{|c|c|c|c|c|c|c|}
\hline & \multicolumn{4}{|c|}{ Resultado del parto } & \multirow{2}{*}{\multicolumn{2}{|c|}{ Total }} \\
\hline & \multicolumn{2}{|c|}{ Adecuado } & \multicolumn{2}{|c|}{ Inadecuado } & & \\
\hline & $\mathbf{n}$ & $\%$ & $\mathbf{n}$ & $\%$ & $\mathbf{n}$ & $\%$ \\
\hline \multicolumn{7}{|l|}{$\begin{array}{c}\text { Edad gestacional } \\
\text { (semanas) }\end{array}$} \\
\hline 31 a 36 & 0 & 0,0 & 14 & 8,5 & 14 & 4,1 \\
\hline 37 a 41 & 174 & 100 & 150 & 91,5 & 324 & 95,9 \\
\hline \multicolumn{7}{|l|}{ Líquido amniótico } \\
\hline Claro & 174 & 100 & 81 & 49,4 & 255 & 75,4 \\
\hline Meconiado & 0 & 0,0 & 83 & 50,6 & 83 & 24,6 \\
\hline \multicolumn{7}{|l|}{ Desgarro perineal } \\
\hline Sin desgarro & 174 & 100 & 139 & 84,8 & 313 & 92,6 \\
\hline Primer grado & 0 & 0,0 & 24 & 14,6 & 24 & 7,1 \\
\hline Segundo grado & 0 & 0,0 & 1 & 0,6 & 1 & 0,3 \\
\hline \multicolumn{7}{|l|}{$\begin{array}{c}\text { Índice de Apgar al } \\
\text { minuto }\end{array}$} \\
\hline 7 & 0 & 0,0 & 3 & 1,8 & 3 & 0,9 \\
\hline 8 & 10 & 5,7 & 36 & 22,0 & 46 & 13,6 \\
\hline 9 & 164 & 94,3 & 125 & 76,2 & 289 & 85,5 \\
\hline \multicolumn{7}{|c|}{ Peso recién nacido $(\mathrm{g})$} \\
\hline 1950 a 2500 & 0 & 0,0 & 9 & 5,5 & 9 & 2,7 \\
\hline 2501 a 3999 & 174 & 100 & 112 & 68,3 & 286 & 84,6 \\
\hline 4000 a 4360 & 0 & 0,0 & 43 & 26,2 & 43 & 12,7 \\
\hline Total & 174 & 100 & 164 & 100 & 338 & 100 \\
\hline
\end{tabular}

Fuente: Autor 
Tabla 4. Características sociales, reproductivas y obstétricas asociadas al resultado adecuado del parto

\begin{tabular}{|c|c|c|c|c|}
\hline & \multicolumn{2}{|c|}{ Resultado del parto } & \multirow{4}{*}{$\begin{array}{l}\text { OR crudo } \\
\text { (IC95\%) }\end{array}$} & \multirow{4}{*}{$\begin{array}{l}\text { OR ajustado' } \\
\text { (IC95\%) }\end{array}$} \\
\hline & Adecuado & Inadecuado & & \\
\hline & $(n=174)$ & $(n=164)$ & & \\
\hline & $\%$ & $\%$ & & \\
\hline Parto & 51,6 & 48,4 & & \\
\hline \multicolumn{5}{|l|}{ Características biosociales } \\
\hline Edad (20 a 35 años) & & & $p=0,009$ & $p=0,002$ \\
\hline Sí & 73,0 & 84,8 & $0,5(0,3-0,8)$ & $0,4(0,2-0,7)$ \\
\hline No & 27,0 & 15,2 & 1,0 & 1,0 \\
\hline Conviviente/casada & & & $p=0,871$ & \\
\hline Sí & 96,0 & 96,3 & $0,9(0,3-2,8)$ & - \\
\hline No & 4,0 & 3,7 & 1,0 & \\
\hline Nivel educativo secundario o superior & & & $p=0,028$ & $p=0,025$ \\
\hline Sí & 97,7 & 92,7 & $3,4(1,1-10,8)$ & $4,1(1,2-13,8)$ \\
\hline No & 2,3 & 7,3 & 1,0 & 1,0 \\
\hline Ama de casa & & & $p=0,886$ & \\
\hline No & 56,9 & 57,9 & $1,0(0,6-1,5)$ & - \\
\hline Sí & 43,1 & 42,1 & 1,0 & \\
\hline \multicolumn{5}{|l|}{ Características reproductivas } \\
\hline Menarquia & & & $p=0,825$ & \\
\hline$<12$ años & 86,2 & 85,4 & $1,1(0,6-2,0)$ & - \\
\hline$\geq 13$ años & 13,8 & 14,6 & 1,0 & \\
\hline Uso de método anticonceptivo & & & $p=0,280$ & \\
\hline Sí & 73,0 & 78,0 & $0,8(0,5-1,3)$ & - \\
\hline No & 27,0 & 22,0 & 1,0 & \\
\hline \multicolumn{5}{|l|}{ Características obstétricas } \\
\hline Control prenatal & & & $p=0,005$ & $p=0,007$ \\
\hline Más de cuatro & 93,7 & 84,1 & $2,8(1,3-5,9)$ & $2,9(1,4-6,3)$ \\
\hline Menos o igual a cuatro & 6,3 & 15,9 & 1,0 & 1,0 \\
\hline Antecedente de aborto & & & $p=0,561$ & \\
\hline No & 73,6 & 70,7 & $1,2(0,7-1,9)$ & - \\
\hline Sí & 26,4 & 29,3 & 1,0 & \\
\hline Antecedente de parto & & & $p=0,126$ & \\
\hline Sí & 90,8 & 95,1 & $0,5(0,2-1,2)$ & - \\
\hline No & 9,2 & 4,9 & 1,0 & \\
\hline Psicoprofilaxis obstétrica & & & $p=0,006$ & $p=0,034$ \\
\hline Sí & 44,3 & 29,9 & $1,9(1,2-2,9)$ & $1,7(1,03-2,7)$ \\
\hline No & 55,7 & 70,1 & 1,0 & 1,0 \\
\hline Apoyo del acompañante & & & $p=0,833$ & \\
\hline Sí & 22,4 & 21,3 & $1,1(0,6-1,8)$ & - \\
\hline No & 77,6 & 78,7 & 1,0 & \\
\hline Total & 100,0 & 100,0 & & \\
\hline
\end{tabular}

'porcentaje global $=61,0 \%$ prueba de Hosmer y Lemeshow $=1,209 p=0,877 \mathrm{r} 2$ de cox y snell $=0,081$

Fuente: Autor. 


\section{Discusión}

Resulta importante señalar que el 51,6\% de los partos presentaron resultado adecuado, esto muestra los esfuerzos que hace el sistema de salud para poder llegar a buen término, también resulta necesario indicar que posiblemente una gestante que presente un problema de salud, ya sea en su esfera biosocial, presentará también problemas obstétricos o de otra índole, por ello casi la mitad tuvieron resultados inadecuados del parto. Entre los factores que se han podido asociar desde el punto de vista biosocial se encuentra la edad y el nivel educativo, es posible que en las adolescentes y gestantes de edad materna avanzada se cuenta con mayor vigilancia en la atención prenatal, preparación psicoprofiláctica del parto y cuidados en la sala de partos (por las condiciones que trae una gestación en esas edades), por otro lado, Amaya et al, ${ }^{21}$ identificaron que el ser adolescente fue un factor protector que disminuyó el riesgo de terminación del parto por cesárea (RR 0,85 IC $95 \%$ 0,78 - 0,93) y preeclampsia (RR 0,65; IC $95 \%$ $0,46-0,91)$.

En Perú, los indicadores educativos muestran que cada vez es mayor el número de mujeres que acceden a la educación superior, razón por la cual el $51,6 \%$ presentó nivel educativo secundario y un $43,7 \%$ nivel superior, además las pacientes atendidas por el seguro social de EsSalud son en su mayoría trabajadoras afiliadas al seguro o beneficiaras de trabajadores formales, por ello hay mayor probabilidad que su nivel educativo sea secundario o superior, puesto que vienen de empresas formales. Abril-Gonzáles et al, ${ }^{9}$ en su estudio sobre partos con desgarro perineal encontraron que el $79,3 \%$ de las que atendieron tenían una escolaridad secundaria, técnica o universitaria. Ávila et $a l,{ }^{22}$ en su estudio sobre recién nacidos macrosómicos y recién nacidos normales, concluyeron que en los niños de peso normal, el $72 \%$ de las madres tenían escolaridad secundaria, preparatoria o profesional. Las gestantes con mejor nivel educativo, comprenden mejor las explicaciones y el proceso de la gestación, y por ende, tienen mayores resultados adecuados del parto.

El punto de corte para determinar un resultado adecuado del parto se ha presentado en gestantes que han tenido cuatro o más controles prenatales, lo cual puede reflejar que la gestación en términos generales es normal, es decir, que este número de controles resulta apropiado para la gestante que tendrá un curso normal del embarazo, parto y posparto. Un informe realizado por Villar et al, ${ }^{23}$ encontró que la reducción en el número de consultas para el control prenatal no necesariamente produjo un aumento de los resultados biológicos adversos en la madre o el neonato, pero si recalcó que es posible que las mujeres estén menos satisfechas con la reducción en el número de consultas. La norma técnica de salud para la atención integral de salud materna ${ }^{12}$, define a la gestante controlada aquella que ha cumplido con seis controles prenatales, lo que refleja que para la obtención de un resultado adecuado del parto no serían seis controles prenatales, sino un mínimo de cuatro, que es la propuesta de Villar et al,,23 más apropiado en gestantes que cursan un embarazo y parto normal, pero no sería lo apropiado con gestantes de riesgo.

Otro elemento que contribuye en la salud materna y perinatal es lo correspondiente a la psicoprofilaxis obstétrica ${ }^{16}$, en este estudio se observó que existe 1,9 veces más de probabilidad de tener un resultado adecuado del parto si se acude a las sesiones de psicoprofilaxis. Chable-Uitz y García-Cruz ${ }^{24}$ en su estudio con 254 gestantes, concluyeron que esta herramienta logra que las gestantes participen activamente en el parto controlando su temor y dolor. Otro estudio ${ }^{15}$ encontró que las que habían llevado el curso hasta después del parto $(96,4 \%)$ se sentían seguras y satisfechas de haber recibido clases y se comprobó que el conocimiento adquirido en el curso contribuyó a una buena labor de parto, pues este porcentaje de embarazadas refirieron que se acordaron de todas las clases durante el trabajo de parto. Acevedo-Caballero y Solano-Avendaño ${ }^{14}$ en un estudio con 40 mujeres embarazadas entre los 16 a 38 años que habían contado con psicoprofilaxis obstétrica, encontraron que el $75 \%$ mejoró su capacidad ventilatoria en el parto y el $85 \%$ terminó su parto por vía vaginal. $\mathrm{Gazca}^{13}$ en su estudio realizado después del parto, encontró que 19 de 20 gestantes consideraron a la preparación en psicoprofilaxis como útil.

La convivencia y la ocupación ama de casa, no se asociaron al resultado adecuado del parto, estas variables biosociales posiblemente ya no estén repercutiendo apropiadamente en los desenlaces maternos. Ávila et a ${ }^{22}$ encontraron que el $59 \%$ de las mujeres con recién nacidos macrosómicos estaban en unión libre o convivencia, el $86 \%$ manifestó trabajar en labores del hogar; similar a lo encontrado 
para las madres de recién nacidos normales (56\%) y aquellas que eran amas de casa (94\%).

Osorio et al,7 en su estudio sobre factores de riesgo para mortalidad neonatal precoz, encontró que en el grupo de neonatos que fallecieron, el 93,8\% eran madre ama de casa y 33,3\% estaban en unión libre; parecido a aquellos recién nacidos vivos con madres que desempeñaban la misma labor $(93,8 \%)$ y tenían una unión libre (25\%). Las variables reproductivas como la menarquia precoz y el uso de método anticonceptivo previo al embarazo tampoco presentaron asociación. También es conveniente indicar que en estas variables los resultados son similares entre las que presentan resultado adecuado e inadecuado del parto por lo que no se ha podido identificar marcadamente diferencias en estos grupos, esto podría deberse a efectos del tamaño de la muestra. Se considera también que este por ser un estudio trasversal, mantiene las dificultades de este diseño, faltarían estudios longitudinales prospectivos que puedan reforzar estos hallazgos.

Finalmente el apoyo del acompañante tampoco resultó asociado, Hodnett et $a^{25}$, realizaron una revisión sistemática que incluyó a 12791 mujeres, y concluyeron que las que recibieron apoyo continuo intraparto tuvieron un 10\% menos probabilidad de parto por cesárea, requirieron menos analgesia, menor uso de instrumentos en el parto y manifestaron mayor satisfacción con la experiencia del parto. Gómez y et $a^{26}$ indicaron en su estudio que el acompañamiento continuo a la mujer durante el trabajo de parto y el parto por un familiar u otra persona, son prácticas benéficas, además este mismo autor sostiene que en América Latina desafortunadamente casi la mitad de las mujeres no tienen acompañamiento durante su trabajo de parto. En este estudio tanto en las de resultado adecuado del parto como inadecuado, $77,6 \%$ y $78,7 \%$ respectivamente, no se practicó el parto con acompañante, lo que llevaría a que las gestantes son atendidas con aislamiento del parto en el hospital de un familiar o pareja.

\section{Conclusiones}

Alrededor de la mitad de las gestantes presentan resultado adecuado del parto, las características biosociales como la edad entre 20 a 35 años y el nivel educativo superior se asociaron a un resultado adecuado del parto. Asímismo, las características obstétricas como el control prenatal y la psicoprofilaxis obstétrica también se relacionaron al mismo resultado. No se encontró asociación con características reproductivas.

\section{AgradeCIMIentos}

A la bachiller en Obstetricia Merly Ventura Rojas Facultad de Ciencias de la Salud Filial Ica Universidad Alas Peruanas por su apoyo en la recolección de información.

\section{Financiamiento}

La presente investigación fue autofinanciada.

\section{CONFLICTO DE INTERÉS}

El autor manifiesta no tener conflicto de intereses

\section{Referencias Biblográficas}

1. Alcalde-Rabanal JE, Lazo-González O, Nigenda G. Sistema de salud de Perú. Salud Publica Mex. 2011;53 supl 2:243-254.

2. Instituto Nacional de Estadística e Informática Perú. Encuesta Demográfica y de Salud Familiar Nacional y Departamental. Perú; 2012.

3. Dirección Regional de Salud de Ica. Análisis de la situación de salud de la región Ica 2011. Oficina de Epidemiología; 2011.

4. Arnau J, Martínez E, Vigueras D, Bas E, Morales R, Álvarez L. Los conceptos del parto normal, natural y humanizado. El caso del área I de salud de la región de Murcia. Rev Antrop Iberoamer. 2012;7(2):225-247.

5. Menéndez G, Navas I, Hidalgo Y, Espert J. El embarazo y sus complicaciones en la madre adolescente. Rev Cubana Obstet Ginecol. 2012;38(3):333-342.

6. Heras B, Gobernado J, Mora P, Almaraz A. La edad materna como factor de riesgo obstétrico. Resultados perinatales en gestantes de edad avanzada. Prog Obstet Ginecol. 2011;54(11):575-580.

7. Osorio-Amézquita C, Romero-Vázquez A. Factores de riesgo asociados a mortalidad neonatal precoz. Hospital general "Dr. Gustavo A. Rovirosa Pérez”, Tabasco, 2005. Salud Tabasco. 2008;14(1-2):721-726

8. Chávez-Courtois M, Casanueva E. Uso de servicios preventivos de salud materno-infantil en un grupo de mujeres mexicanas. Rev Salud Publica. 2005;7(1):15-25.

9. Abril-González F, Guevara-Villarreal A, Ramos-Cruz A, RubioRomero J. Factores de riesgo para desgarro perineal en partos sin episiotomía atendidos por personal en entrenamiento en un hospital universitario de Bogotá (Colombia) 2007. Rev Colombiana Obstet Ginecol. 2009;60(2):143-151.

10. Alfaro N, Campos G. Análisis del control prenatal que se brinda a las gestantes de la provincia de Heredia que tuvieron su parto en el Hospital San Vicente de Paúl. Enf Actual Costa Rica. 2014;26:1-19.

11. Villar J, Bergsjo P. Modelo de control prenatal de la OMS. Grupo de Investigación del Estudio de Control Prenatal de la OMS. Ginebra: Organización Mundial de la Salud, 2003.

12. Ministerio de Salud del Perú. Norma técnica de Salud para la atención integral de salud materna. Resolución Ministerial N 827 2013/MINSA del 24 de diciembre de 2013.

13. Gazca J. Parto indoloro psicoprofiláctico. 20 observaciones personales. Ginecol Obstet Mex. 2014;82:210-22.

14. Acevedo-Caballero J, Solano-Avedaño G. Parto psicoprofiláctico 
en gestantes que asisten a la clínica de especialidades de la mujer. Rev Sanid Milit Mex. 2006;60(5):319-23.

15. Vidal A, Reyes M, Fernández Y, González G. Preparación psicofísica para el parto: seguridad y satisfacción a las gestantes. MediSur 2012;10(1):17-21

16. Ministerio de Salud del Perú. Guía Técnica para la Psicoprofilaxis Obstétrica y Estimulación Prenatal. Resolución Ministerial N ${ }^{\circ} 361$ 2011/MINSA del 6 de mayo de 2011.

17. Cantero A, Fiuri L, Furfaro K, Jankovic P, Llompart V, San Martín E. Acompañamiento en sala de partos: regla o excepción. Rev. Hosp. Mat. Inf. Ramón Sardá 2010;29(3):108-12.

18. Nápoles D, Couto D, Montes de Oca A. Modalidades terapéuticas en la fase latente prolongada del trabajo de parto. MEDISAN 2012;16(5):736

19. Mongrut A. Tratado de Obstetricia. $3^{\circ}$ Ed. Tomo I. Lima, Perú: serator; 1989.

20. Pérez S, Donoso S. Obstetricia. $2^{\text {a }}$ Ed. Santiago de Chile. Edit Mediterráneo; 1992.
21. Amaya J, Borrero C, Ucrós S. Estudio analítico del resultado del embarazo en adolescentes y mujeres de 20 a 29 años en Bogotá. Rev Colomb Obstet Ginecol. 2005;56(3):216-24.

22. Ávila R, Herrera M, Salazar C, Camacho R. Factores de riesgo del recién nacido macrosómico. Pediatr Mex. 2013;15(1):6-11.

23. Villar J, Carroli G, Khan D, Piaggio G, Gülmezoglu M. Patrones de control prenatal de rutina para embarazos de bajo riesgo (Revisión). The Cochrane Library traducida. 2007;4.

24. Chable-Uitz G, García-Cruz K. Ventajas de la psicoprofilaxis perinatal. Rev Sanid Milit Mex. 2013;67(3):106-14

25. Hodnett E, Gates S, Hofmeyr G, Sakala C. Apoyo continuo para las mujeres durante el parto. La Biblioteca Cochrane Plus; 2005 Número 3. Oxford: Update Software Ltd. Disponible en: http:// www.update-software.com

26. Gómez J, Londoño J, Monterrosa E. Frecuencia de uso de prácticas médicas basadas en la evidencia en el cuidado del parto en hospitales de la ciudad de Medellín, años 2004-2005. Iatreia. 2006;19(1):5-13. 